\title{
Comptonisation and Time-lags in Multi-temperature Plasmas Surrounding Compact Objects
}

\author{
Jason Cullen ${ }^{1,2}$ \\ ${ }^{1}$ Special Research Centre for Theoretical Astrophysics, School of Physics, \\ University of Sydney, Sydney, NSW 2006, Australia \\ cullen@physics.usyd.edu.au \\ ${ }^{2}$ Sir Frank Packer Department of Theoretical Physics, School of Physics, \\ University of Sydney, Sydney, NSW 2006, Australia
}

Received 1999 May 11, accepted 2000 January 11

\begin{abstract}
This paper investigates a recent model proposed by Moskalenko, Collmar \& Schönfelder (1998) for the plasma surrounding galactic black hole candidates. Using a linear Monte Carlo code, the spectral and temporal behaviour of the model is found for the case where the plasma geometry consists of a hot shell surrounding a cooler spherical core. The spectrum produced by this model at X-ray and gamma-ray energies is obtained numerically. Also found for the first time in this geometry are the photon time-lags between two energy bands due to rapid aperiodic variability. It is argued that the time-lag information may be able to determine whether this particular geometry is a realistic model for the material surrounding galactic black hole candidates.
\end{abstract}

Keywords: black hole physics — X-rays: stars — plasmas — gamma-rays: theory radiative transfer - accretion

\section{Introduction}

Galactic black hole candidates (BHCs) are known to be sources of high energy photons. This emission is thought to be powered by the gravitational potential energy release resulting from material accreting onto a compact object, by either wind accretion or Rochelobe overflow from a companion. The spectrum of galactic BHCs is often described as being in either a 'high' state, or a 'low' state, according to the flux in the $2-10 \mathrm{keV}$ band. The high state spectrum consists of a blackbody component of temperature $\sim 1 \mathrm{keV}$ and a power law of photon spectral index $\sim 2 \cdot 2-2 \cdot 7$, while the low state spectrum consists of a single power law of photon spectral index $\sim 1.7$ (Esin et al. 1998; Tanaka \& Lewin 1996).

This power-law component is attributed to blackbody photons being up-scattered by electrons (that is, the photons gain energy from the electrons via the inverse Compton process) as they traverse a corona of hot plasma with a Maxwellian electron temperature 10-100 keV (Sunyaev \& Titarchuk 1980; Bisnovatyi-Kogan \& Blinnikov 1976). If the Comptonisation is saturated, where the photons are in thermal equilibrium with the electrons, then the cut-off in the spectrum occurs at $\sim 3 k T_{e}$, where $k T_{e}$ is the temperature of the coronal electrons. However, the COMPTEL instrument on the Compton Gamma-Ray Observatory satellite has detected significant gamma-ray emission up to energies of several $\mathrm{MeV}$, which cannot be explained in this canonical
Comptonisation model because the gamma-ray flux is too high for what are thought to be reasonable values of the coronal temperature.

While inverse Compton (IC) scattering is understood to be one of the important processes for producing high energy photons from galactic black hole candidates (as well as accreting neutron stars and active galactic nuclei), the physical geometry of the coronal plasma responsible for scattering the photons is not well constrained by current observations. This is partly due to the nature of IC scattering: in general, it depends only on the temperature and the optical depth of the plasma, rather than depending on the physical dimensions directly.

The two geometries that are commonly assumed for spectral calculations are the plane-parallel 'slab' corona above a standard thin accretion disk, and a spherical or quasi-spherical corona surrounding the innermost part of an accretion disk. Based on the results of non-linear Monte Carlo spectral simulations (Dove et al. 1997), the spherical corona plus disk geometry best accounts for the low (hard) state spectrum of galactic black hole candidates. For example, Cyg X-1 spends most of its time in the low state.

The problem then remains of how to produce gamma-ray photons. Possibilities that have been considered are a non-linear tail to the electron Maxwellian distribution, which would be generated

$10 \cdot 1071 /$ AS00003 1323-3580/00/010048 $\$ 05.00$ 
by some magnetohydrodynamic (MHD) process, or a compact electron-positron pair dominated plasma. It has been pointed out that the latter possibility is unlikely (Moskalenko, Collmar \& Schönfelder 1998; hereafter MCS), as the luminosity of the gamma rays, although small, still exceeds the Eddington limit for a pair plasma.

Recently MCS have suggested that a twocomponent corona model can explain both the X-ray and gamma-ray emission of BHCs such as Cyg X-1 (see Figure 1). In this model, the plasma geometry consists of two concentric spheres of plasma, each with a different temperature and optical depth. The compact spherical inner-corona of Thompson optical depth of approximately $\tau \simeq 1 \cdot 4-2 \cdot 4$ is responsible for the X-ray power-law component, while a tenuous extended spherical outer-corona of $\tau \simeq 0.05$ consists of relativistic electrons that are responsible for the hard gamma-ray tail.

\section{Schematic diagram of the proposed plasma geometry surrounding GBHCs}

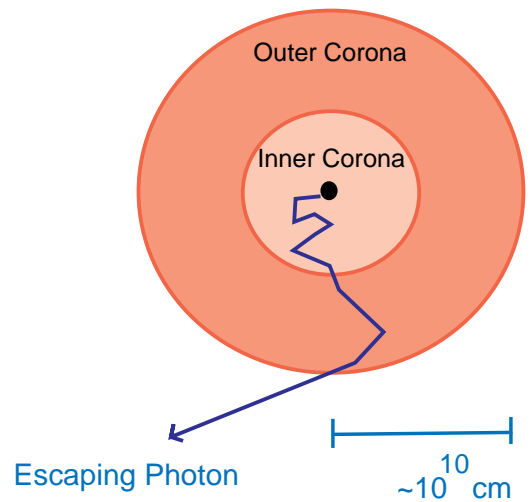

Figure 1-Schematic diagram of the plasma geometry surrounding galactic BHCs as considered in this paper. We consider a model in which an inner spherical corona of plasma is surrounded by an outer spherical corona of hotter, tenuous plasma. Photons emitted in the central regions (taken to be a point source) gain energy from the electrons in these coronae by inverse Compton scattering. The inner optically thick corona produces the canonical X-ray powerlaw, while the outer corona scatters some of the escaping photons up to gamma-ray energies. The time-lags between different energy bands (due to different photon escape times from the cloud) can then be found by summing over the pathlength travelled by the escaping photons.

The acceleration mechanism for the outer-corona electrons is unknown, but could be any one of stochastic acceleration, MHD turbulence, plasma instabilities in the inner-corona or accretion disk, or an outflow of electron-positron pairs that are created close to the central object. The outer-coronal density is low, so electron cooling is inefficient and the temperature is high $\simeq 400 \mathrm{keV}$. MCS estimated that the cooling time of these electrons due to IC scattering is a few seconds, so the outer-coronal electrons are effectively confined to a region a few light seconds across (at most).

It has also been emphasised (Kazanas, Hua \& Titarchuk 1997) that any realistic model for a corona in galactic BHCs must also account for their temporal behaviour, such as the hard X-ray time-lags, as well as the spectral fit. These time-lags are produced as photons diffuse through the corona. The photons that scatter numerous times are delayed with respect to those photons that escape after undergoing few scatterings. Photons that scatter many times on average gain more energy than the other photons and so the high energy photons are delayed (take longer to escape from the plasma cloud) when compared to lower energy photons.

As recent investigations have shown (Kazanas, Hua \& Titarchuk 1997; Böttcher \& Liang 1998), this time delay or lag between high and low energy photons is sensitive to the exact geometry of the up-scattering plasma, including density gradients, and so provides us with a tool that can in principle determine the geometry of the coronal plasma in galactic BHC systems.

We investigate using a linear Monte Carlo code whether the model suggested by MCS can explain both the X-ray and gamma-ray emission of BHCs. We obtain the spectrum produced by such a model for the 'low' state where IC is the dominant process. Furthermore, we also calculate the photon time-lags between the energy bands $2-10 \mathrm{keV}$ and $0 \cdot 01-10$ $\mathrm{MeV}$.

\section{Algorithm of the MONTE CARLO Simulation}

Our Monte Carlo code is based on the standard algorithm given in Pozdnyakov, Sobol' \& Sunyaev (1983). It simulates inverse Compton scattering of photons off relativistic Maxwellian electrons in a plasma of arbitrary electron temperature and optical depth. The scattering probability and the meanfree-path of the photons is determined according to the prescription in Pozdnyakov, Sobol' and Sunyaev (1983) for relativistic Maxwellian electrons. We consider a spherically symmetric case, with the source of photons at the centre of a plasma cloud. The plasma cloud is divided into a shell and a spherical core, each with different temperatures and optical depths (Figure 1). The cloud is illuminated by a central source of blackbody photons with injection taken to be a delta function in time. In the simulations we fix the outermost radius of the cloud to be $1 \cdot 0$ light second across.

When a photon crosses the boundary from the inner to the outer corona it is brought back to the boundary, and (without changing its propagation direction) a new mean free path is calculated based on the temperature and optical depth of the outer corona. After the photon has entered the outer cloud and scattered, a routine will determine whether the 


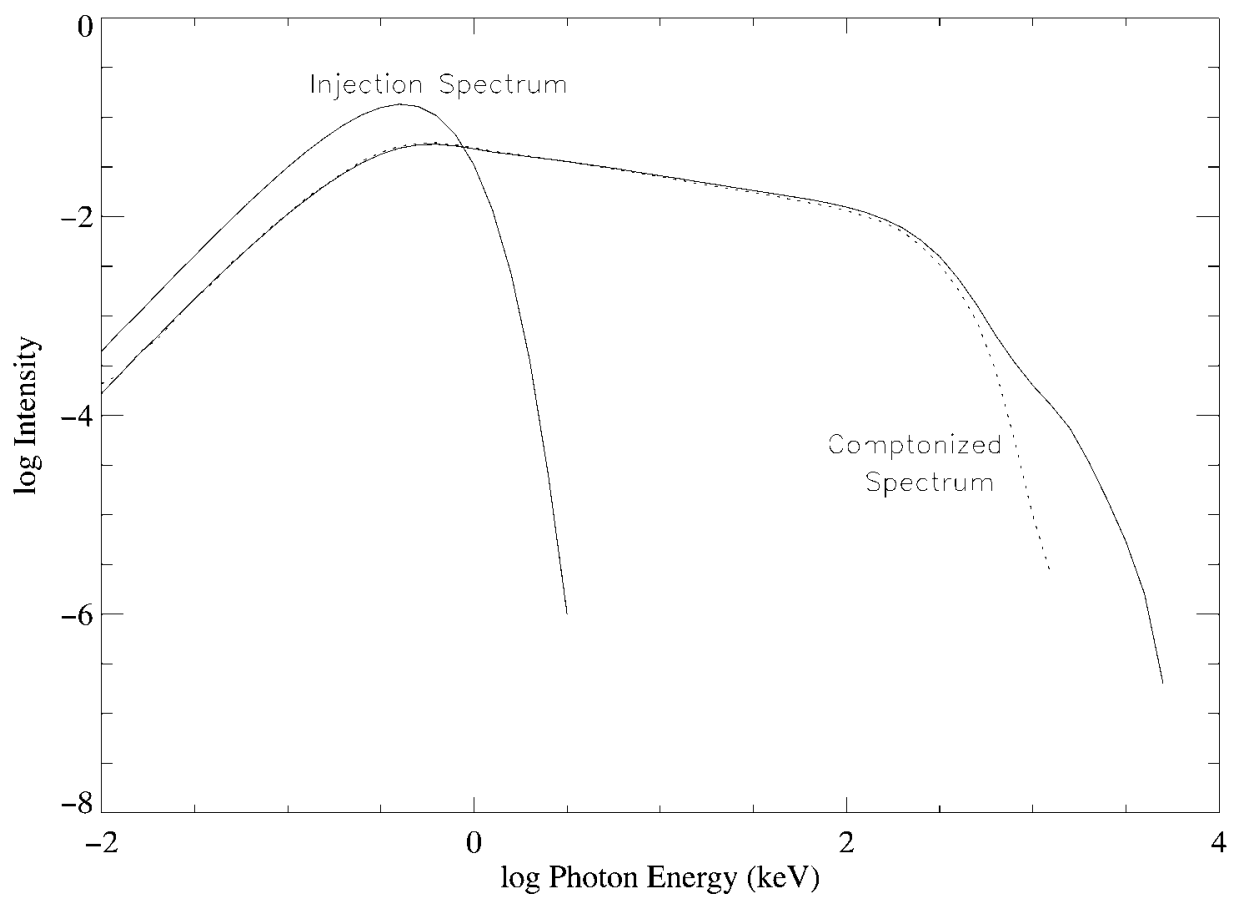

Figure 2-Typical spectrum produced by the MCS model, as well as the injection spectrum and the single-temperature (isothermal) corona spectrum (dotted line, for comparison). The injected spectrum is a blackbody distribution. Photons gain energy by inverse Compton scattering as they diffuse through the cloud, forming a power-law. The single-temperature corona spectrum has a cutoff below $1.0 \mathrm{MeV}$, while the multi-temperature model extends upwards into the MeV range. This high energy excess is due to the photons that are scattered up to gamma-ray energies in the high temperature outer cloud. Note that the X-ray power-law part of the spectrum has the same slope for both the single and double corona models. This is required as the canonical single corona model accounts well for the X-ray part of the spectrum.

photon is heading back towards the inner cloud. Photons that subsequently re-enter the inner corona are again placed on the boundary, and the optical depth and temperature are reset to their values in the inner cloud. Photons that escape from the plasma cloud are binned in energy to produce the observed spectrum.

The pathlength traveled by each photon is tracked and summed to give the total distance traveled (in units normalised to the outer cloud radius). This is converted into an escape time from the cloud which is binned to give the light curves, that is, the intensity in various bands as a function of time. These light curves are therefore Green's functions in the sense that they represent the response of the system to a delta-function source term.

The Fourier time-lags were then produced from the light curves using the FFT routines in IDL. Results were checked with those obtained numerically by Kazanas, Hua \& Titarchuk (1997).

\section{Spectra}

Figure 2 shows the single-temperature (isothermal) coronal Comptonised spectrum produced by a spherical homogeneous cloud of plasma of optical depth $\tau=2 \cdot 39$ and electron temperature of $k T_{e}=76 \cdot 7$ $\mathrm{keV}$ (dotted line). Figure 2 also shows the spectrum produced by assuming the MCS model (solid line).
The inner cloud parameters are the same as in the single cloud case, and the outer cloud is of effective optical depth $\tau=0.06$ and electron temperature $k T_{e}=396 \mathrm{keV}$. These temperatures and optical depths are the best fit values (as determined by MCS) to BATSE-COMPTEL observations of Cyg $\mathrm{X}-1$ taken when it was in the low state. Also shown is the injected source spectrum which is taken to be a blackbody distribution of temperature $130 \mathrm{eV}$. The ratio of the radius of the inner cloud to the outer cloud radius is a free parameter, but the overall energy spectrum is insensitive to this. Here we fix it to be $0 \cdot 1$.

In both cases, photons gain energy by inverse Compton scattering as they diffuse through the cloud, forming a power-law. We see that the single-temperature corona spectrum has a cutoff at below $1.0 \mathrm{MeV}$, while the multi-temperature model produces substantial numbers of photons with energies in the $\mathrm{MeV}$ range. This high energy excess is due to the photons that are scattered up to gamma-ray energies in the high temperature outer cloud.

Note that the X-ray power-law part of the spectrum has the same slope of between $2-100 \mathrm{keV}$ for both the single and double corona models. This is required as the canonical single corona model accounts well for the X-ray part of the spectrum. But while the 


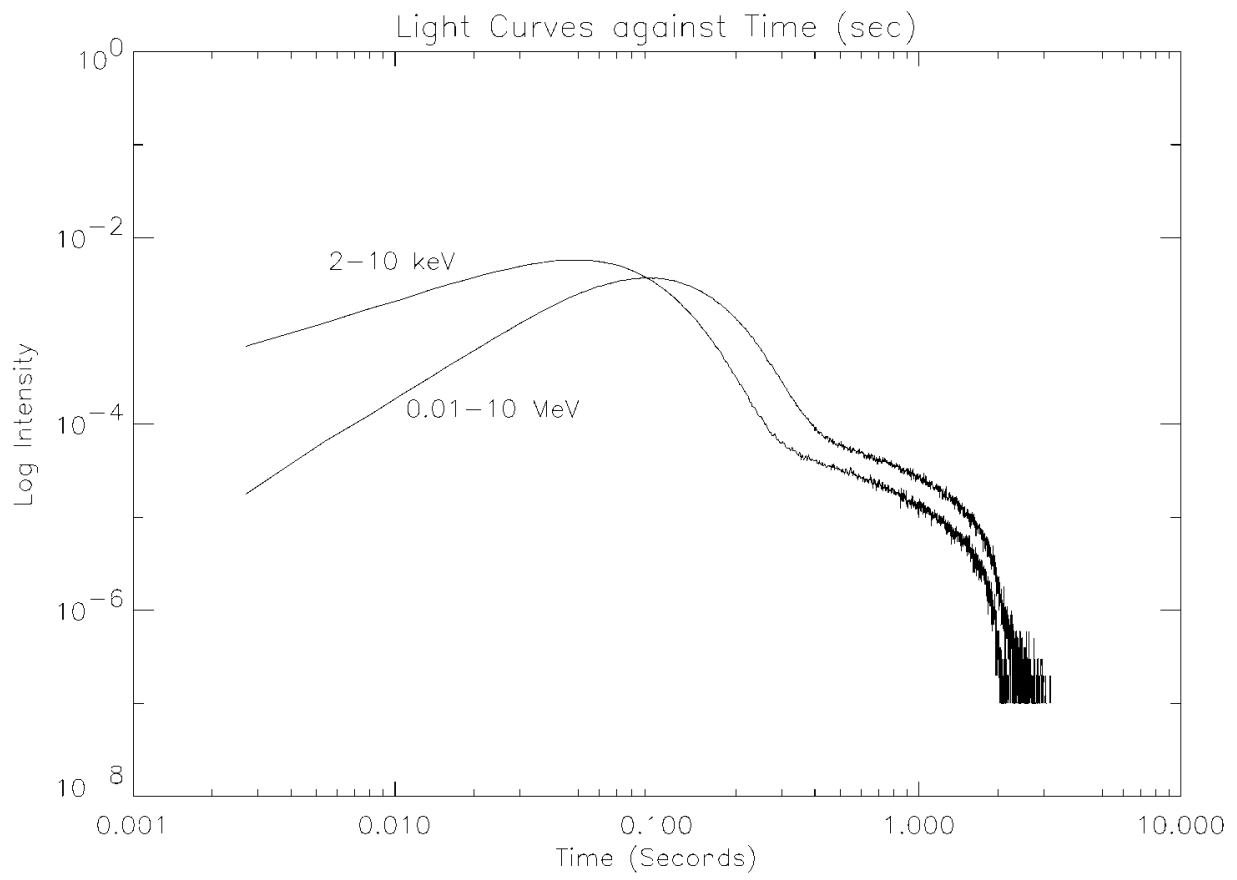

Figure 3 -Intensity in the two X-ray bands against photon escape time. In this graph the inner cloud radius is chosen to be 0.03 light-seconds, while we assume that the physical radius of the outer cloud is $1 \cdot 0$ light-second. The light curves are produced by Comptonisation of a soft photon flare (delta function) at time zero. The cloud parameters are the best fit parameters (taken from MCS) for BATSE-COMPTEL data taken when Cyg X-1 was in the low state. They are: inner cloud $k T_{e}=76 \cdot 7 \mathrm{keV}$ and optical depth $\tau=2 \cdot 39 ;$ outer cloud $k T_{e}=396 \mathrm{keV}$ and optical depth $\tau=0 \cdot 06$.

canonical X-ray power-law is still produced, there is now at high energies an excess of photons above the cutoff energy for a single temperature model. The optical depth of the outer cloud is low enough that the shape of the Comptonised spectrum at low energies is not distorted, however it does act to scatter some photons up to higher energies than they could have reached propagating through a cooler isothermal inner cloud. Therefore, this geometry can potentially explain the production of gamma rays without contradicting inverse Compton models for spectral fits at X-ray energies.

\section{Time-lags}

When IC scattering is the process responsible for producing high energy photons, there will be a hard time-lag between the photons at high and low energies. The photon time-lags between two different energy bands essentially represents the difference in photon pathlength (or escape time) between bands: photons in the higher energy bands tend to have undergone more scattering events, and therefore have traveled further in order to escape the corona.

The time-lags between two energy bands can be found by Fourier transforming the light curves in the high and low energy bands (Miyamoto et al. 1988; van der Klis et al. 1987). The discrete Fourier transforms are then used to form the complex cross power spectrum by multiplying the complex Fourier amplitudes from the low-energy band with the complex-conjugate of the complex
Fourier amplitudes from the high-energy band. The imaginary part of the complex cross power spectrum is then divided by its real part, and the arctangent of the result is taken to find the phase lags between bands. Dividing the phase lags by $2 \pi f$ then gives the hard time-lag curve as a function of Fourier frequency.

Observationally this technique has been used to investigate the time variability of galactic black hole candidates (see e.g. van der Hooft et al. 1999). On the theoretical side, Kazanas, Hua \& Titarchuk (1997) have used this method to investigate the effect of density gradients in the Comptonising plasma, and Böttcher and Liang (1998, 1999) have attempted to determine the location of the photon source with respect to the plasma. It has been found by these authors that the time-lag curve is sensitive to variations in electron density.

In this work we therefore use this method to determine the effect a density discontinuity of the type suggested by MCS has on the time-lag curve. Here we treat the radius of the inner cloud as a free parameter, while the outer radius is fixed at one light-second. Changing the value of the outer radius (for any fixed ratio of the two radii) simply changes the location of the curve in the frequency/time-lag parameter space without changing the shape of the curve. The electron temperature and optical depth of the clouds, as well as the temperature of the blackbody source photons, are the same as in Section 3. 


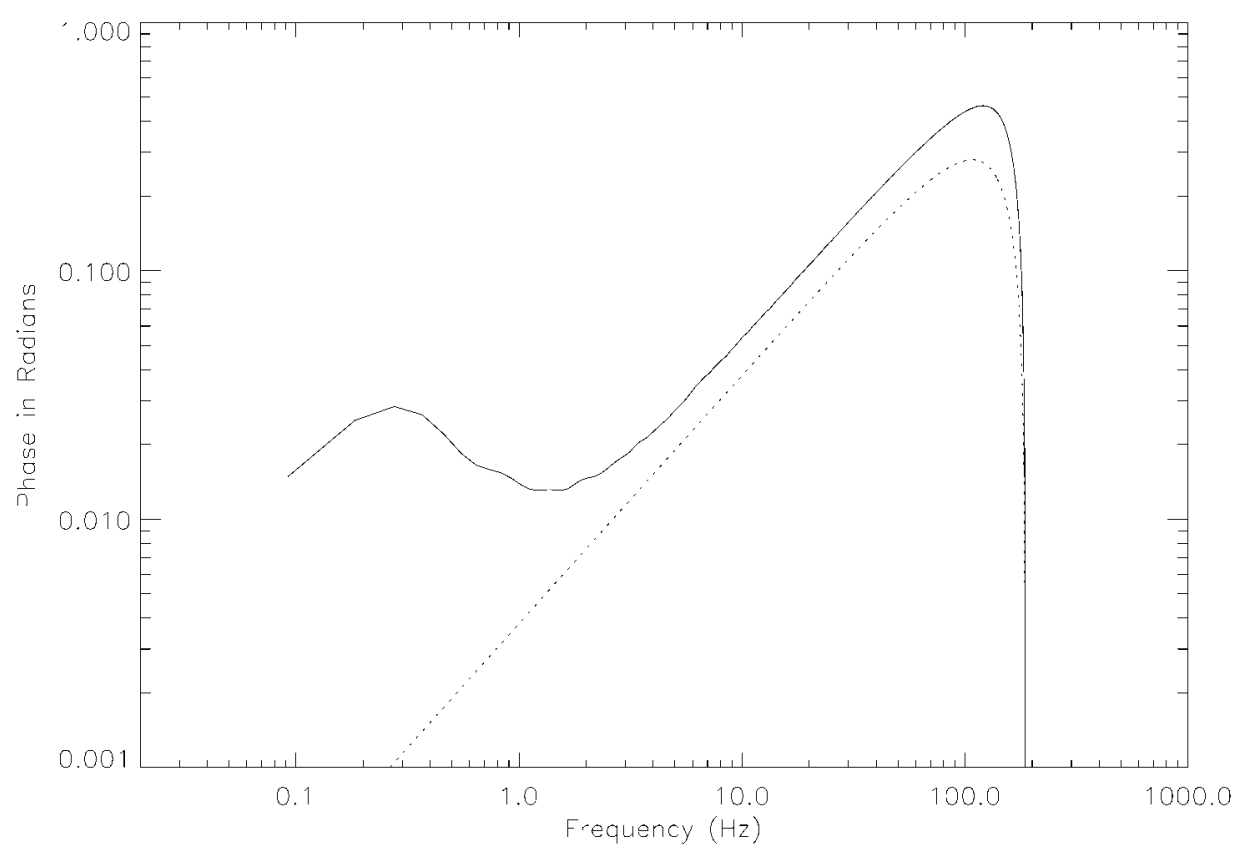

Figure 4-Comparison of the phase lag for two models: the solid curve is the MCS model where the ratio of the inner and outer radii is 0.001 , while the dotted curve is for a homogeneous sphere. In both cases the outer radius is fixed at one light-second. The difference between the two curves at low frequencies is due to the outer shell of plasma, and therefore tells us whether the outer shell exists.

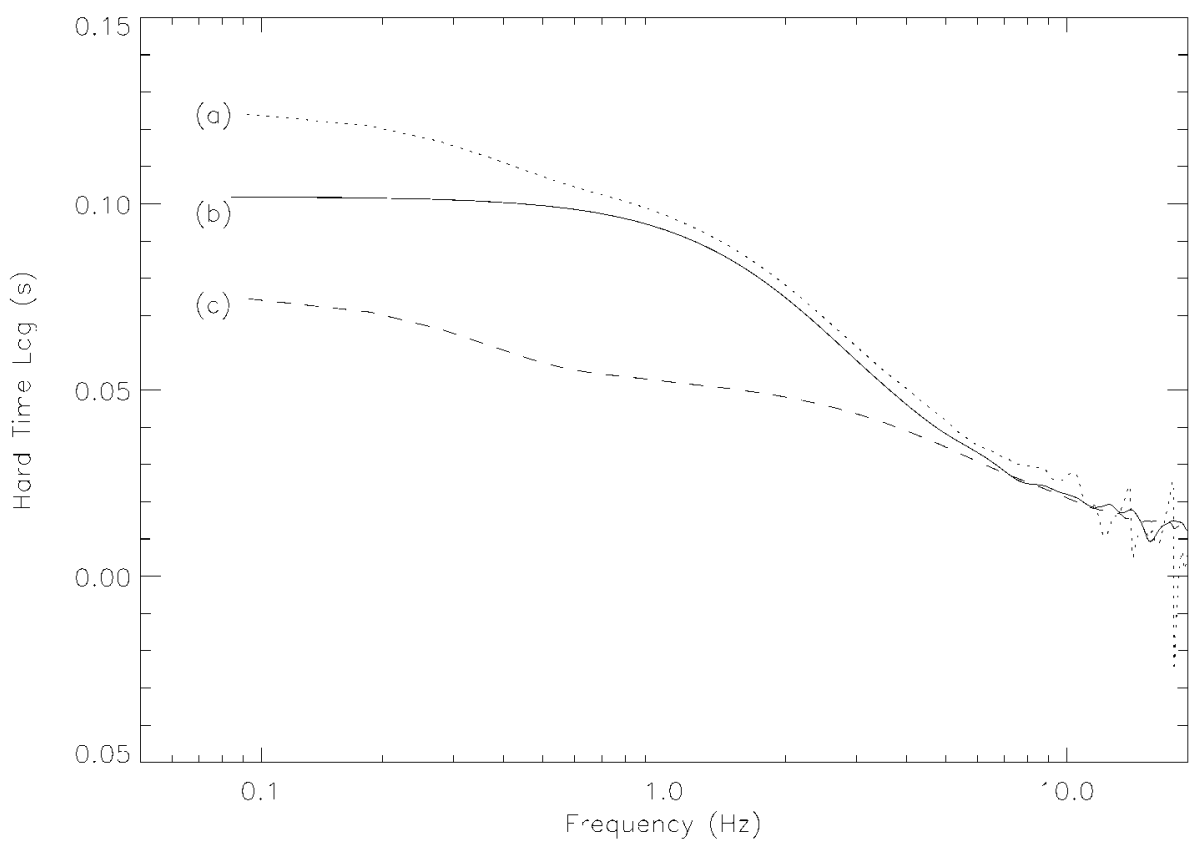

Figure 5-Comparison of time-lags for various values of the ratio of the inner cloud radius to the outer cloud radius for the MCS model. Curve (a) is for the model where the ratio of the inner and outer radii is $0 \cdot 1$, while curve (c) is for a ratio of 0.05. Also shown are the lags due to the inner core only (curve b). The radius of the inner core in that case is taken to be $0 \cdot 1$ light-second. The difference between curves (a) and (b) at low frequencies is due to the outer shell of plasma. 


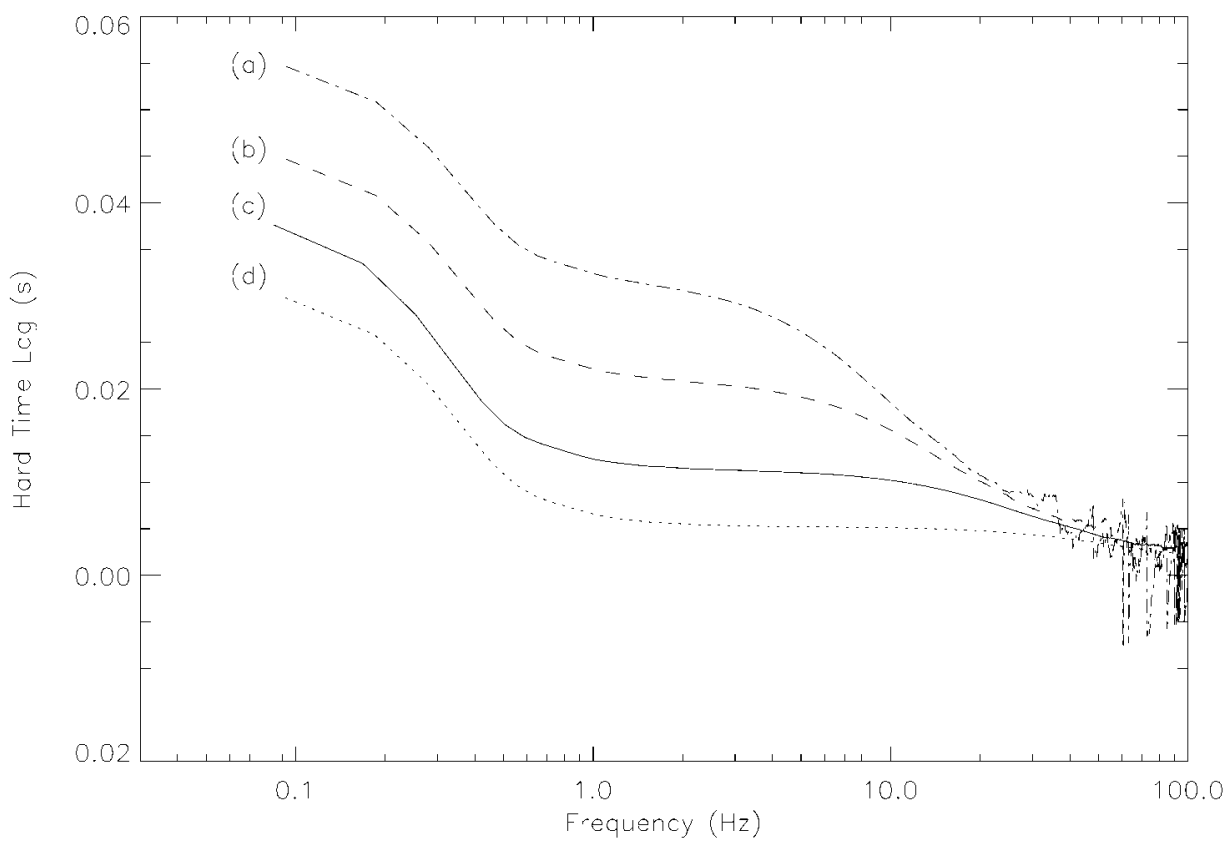

Figure 6-Comparison of time-lags for various small values of the ratio of the inner cloud radius to the outer cloud radius for the MCS model. Curve (a) is for a ratio of 0.03 , curve (b) is for a ratio of 0.02 , curve (c) is for a ratio of $0 \cdot 01$ and curve (d) is for a ratio of $0 \cdot 005$.

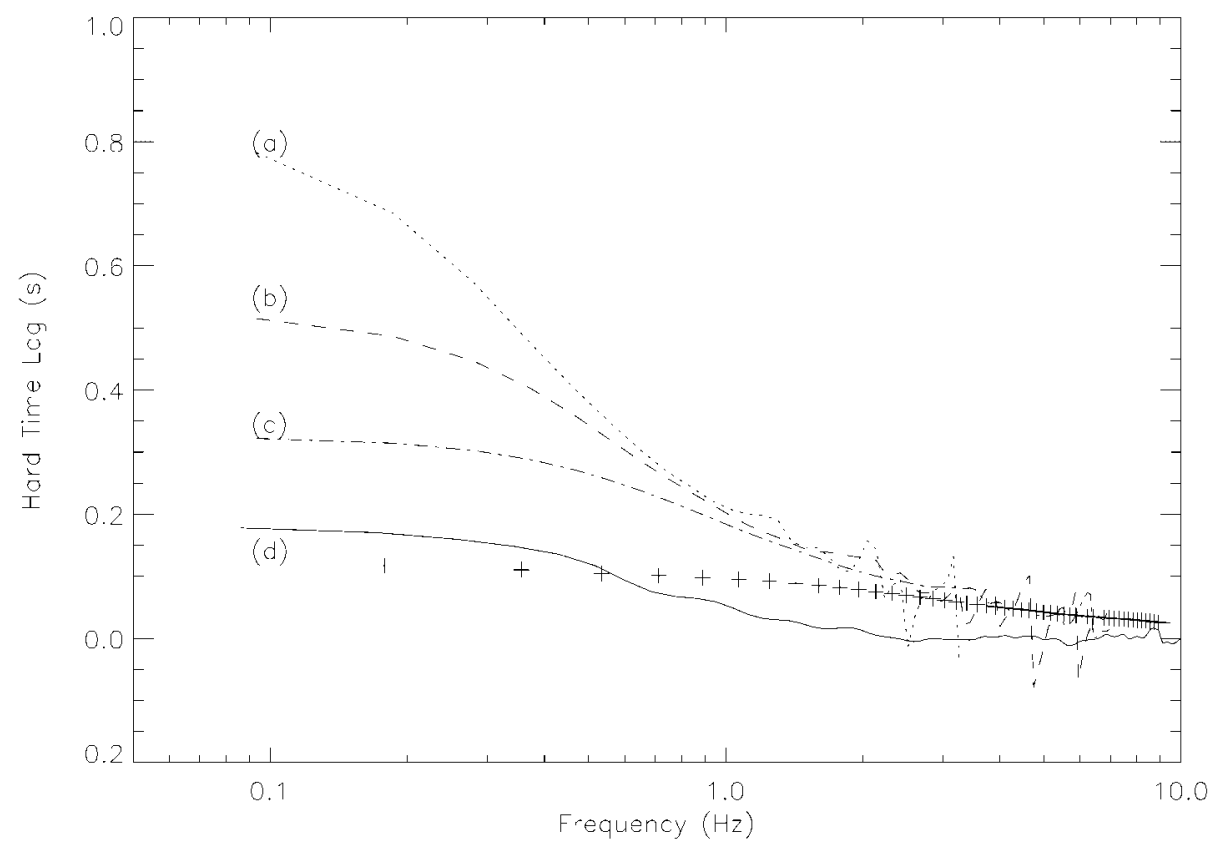

Figure 7-Comparison of time-lags for various large values of the ratio of the inner cloud radius to the outer cloud radius for the MCS geometry. Curve (a) is for a ratio of $0 \cdot 8$, curve (b) for a ratio of $0 \cdot 5$, and curve (c) for a ratio of $0 \cdot 3$. Curve (d) are the lags due to a cloud of radius one light-second with parameters equal to the outer shell plasma in the MCS model. The crosses indicate the lag for a different pair of energy bands (2-10 and 10-400 keV), where the ratio of radii is $0 \cdot 1$. Because these energy bands do not extend to gamma-ray energies no photons from the outer corona contribute to the lags and the curve is flat with no rise at low Fourier frequencies. 
We consider the lags between two different bands, 2-10 keV and $0 \cdot 01-10 \mathrm{MeV}$ (which in this paper we call bands one and two respectively), where we have taken the central illumination of the cloud by the source photons to be an instantaneous delta function in time. Because so few photons scatter in the low optical depth outer cloud, the second band has been made large in order to make the two light curves of comparible intensity.

Figure 3 shows the intensity light curves as a function of time in the two bands, where the inner cloud radius is 0.03 light-seconds. We see that there is a flattening of the light curve at around $0 \cdot 1-0 \cdot 2$ seconds due to photons scattering in the outer cloud. Although the overall spectrum is largely insensitive to the ratio of the inner and outer cloud radii, variations in this ratio do result in different light curves as more or fewer photons scatter in the outer cloud, and therefore result in different hard time-lags when Fourier transformed.

Figure 4 shows the phase lag between bands one and two as a function of Fourier frequency for two different models. The solid curve is the MCS model with temperatures and optical depths as in Section 3. The dotted curve is the phase lag for a homogeneous cloud of plasma with temperature and optical depth equal to that of the inner core of the MCS model. In both cases the outer radius is set to 1 light-second. We see that the solid curve develops a second peak at low frequencies, while the dotted curve is single-peaked. This rise in the solid curve at low frequencies is due to the change in temperature and optical depth in the outer shell of plasma in the MCS model. This therefore indicates the possibility of determining if the MCS geometry exists in BHCs.

Figures 5, 6 and 7 show the hard time-lag curves between bands one and two as a function of Fourier frequency for various values of the inner cloud radius. In Figure 5 for comparison we also show the time-lags due to the inner cloud only (that is, with the outer plasma shell removed). With the outer shell added there is a larger lag at low frequencies than for the inner cloud alone. This is the key difference between simple spherical corona models and the multi-temperature model due to MCS.

Figure 6 shows that for small inner-radius values, the lags become almost constant at high frequencies as the inner radius is made small, but there is still a peak at low frequencies. For large inner-radius values (Figure 7), we are seeing the inner cloud/core only, as fewer photons scatter in the relatively smaller outer cloud/shell. In Figure 7 we also show the lags due to a spherical corona with electron temperature and optical depth equal to that of the outer cloud/shell in the MCS model. This is equivalent to making the inner cloud radius and optical depth approach zero.
The crosses in Figure 7 show the lags for a different pair of energy bands (2-10 and 10-400 $\mathrm{keV}$, where the ratio of radii is $0 \cdot 1)$. Because these bands do not extend up to gamma-ray energies, fewer high energy photons from the outer corona have contributed to the time-lag curve. The lags are therefore almost constant with frequency as we are essentially seeing just the inner corona. In this case the lags tell us little about the outer corona.

The various time-lag curves can now in principle be used to determine the ratio of the inner and outer radii, as well as set the overall scale of the corona. Given the temperatures and optical depths (from spectral modeling) of the inner and outer-coronae, the shape of the hard time-lag curve determines the ratio of the inner and outer radii, while the position of the hard time-lag curve in the frequency/time-lag parameter space determines the magnitude of the outer radius. Thus, if the optical depths and the values of the radii are known, then the density of the corona is known, and we can in principle know all the physical parameters of the corona (assuming the MCS core plus shell model is true).

It is also possible that the outer radius can be found from other criteria, perhaps by identifying the break in the power spectral density of a light curve with the value of the outer radius as suggested by Böttcher \& Liang (1998).

\section{Conclusion}

We have investigated in detail the MCS model for the geometry of the plasma surrounding BHCs. In particular, we have calculated the full spectrum produced by this model using a Monte Carlo simulation, as well as the time dependent light curves produced by Comptonisation of a soft radiation flare at time zero, and the hard time-lag curve between different energy bands. This is the first time the light curves and the hard time-lags from a plasma with the MCS geometry have been investigated. It is found that Monte Carlo investigation of photon time-lags between different energy bands can be used to constrain this multi-temperature geometry for the accretion disk corona around galactic BHCs.

We note that the energy spectrum is largely insensitive to the values of the radii used for the inner and outer clouds, as in general a Comptonised spectrum is sensitive only to the temperature and optical depth of the plasma (and the initial photon distribution if the plasma is optically thin). However, we find that the time-lag curve between energy bands is sensitive to the ratio of the two radii. This is the main result of this work. We find that a sharp rise in the time-lag curve at low Fourier frequencies is the temporal signature of the MCS model.

MCS also considered the case where bremsstrahlung photons from the tenuous outer corona are a significant spectral component. However, if the 
bremsstrahlung photon flux is stationary, then this component will make no contribution to the Fourier transform of the light curves, and therefore no contribution to the time lag curve. This will be the case as long as any fluctuations in the bremsstrahlung flux are small compared to the amplitude of the central Comptonised pulse. Similarly, any time independent processes such as $e^{ \pm}$annihilation should not contribute significantly to the time-lags.

Thus the time delay or lag can be used to determine the overall size of the corona and the ratio of the inner and outer radii, if this two-component corona geometry exists. This information combined with values for the optical depth obtained from spectral modeling can then tell us the overall density of the corona. Such a result would then give us all the physical parameters of the plasma cloud.

We have demonstrated that timing observations at X-ray and gamma-ray energies can in principle determine the physical properties of the two-component corona in the MCS model. It is expected that more zones will be added in future versions of the code. This would enable us to investigate density and temperature gradients in more detail.

\section{Acknowledgments}

I wish to thank Chris Rennie for advice on the IDL routine, and Kinwah $\mathrm{Wu}$ for helpful discussions.

\section{References}

Bisnovatyi-Kogan, G. S., \& Blinnikov, S. I. 1976, Soviet Astron. Lett., 2, 191

Böttcher, M., \& Liang, E. P. 1998, ApJ, 506, 281

Böttcher, M., \& Liang, E. P. 1999, ApJ, 511, L37

Dove, J. B., Wilms, J., Maisack, M., \& Begelman, M. C. 1997, ApJ, 487, 759

Esin, A. A., Narayan, R., Cui, W., Grove, J. E., \& Zhang, S. 1998, ApJ, 505, 854

Kazanas, D., Hua, X. M., \& Titarchuk, L. G. 1997, ApJ, 480,735

Miyamoto, S., Kitamoto, S., Mitsuda, K., \& Dotani, T. 1988, Nature, 336, 450

Moskalenko, I. V., Collmar, W., \& Schönfelder, V. 1998, ApJ, 502, 428

Pozdnyakov, L. A., Sobol', I. M., \& Sunyaev, R. A. 1983, Ap\&SS Reviews, 2, 189

Sunyaev, R. A., \& Titarchuk, L. G. 1980, A\&A, 86, 121

Tanaka, Y., \& Lewin, W. H. G. 1995, in X-Ray Binaries, Cambridge Astrophysics Series, vol. 26, ed. W. H. G. Lewin et al. (Cambridge Univ. Press), p. 126

van der Hooft, F., et al. 1999, ApJ, in press, astro-ph/9810492

van der Klis, M., Hasinger, G., Stella, L., Langmeier, A., van Paradijs, J., \& Lewin, W. H. G. 1987, ApJ, 319, L13 